\title{
RELATIVE STAR POSITIONS FROM OVERLAPPING PHOTOGRAPHIC PLATES
}

\author{
S. V.M. Clube
}

\section{Introduction}

An account is given of an investigation to obtain accurate relative star positions from overlapping photographic plates. The method described is quite general, but in the first instance is to be applied to plate material obtained with the new Cape astrometric camera.

The Cape 4-component $f / 1020-\mathrm{cm}$ astrometric camera produces a field of $5^{\circ}$ square with a scale of $100^{\prime \prime}$ per $\mathrm{mm}$ in the focal plane. The telescope is fitted with a yellow filter $12 \mathrm{~mm}$ from the focal plane, which in combination with the Ilford-R 40 emulsion used, transmits more or less uniformly in the range $5300<\lambda<6500$ and little elsewhere. Within this spectral range, the maximum longitudinal aberration is only $0.75 \mathrm{~mm}$ and the images sensibly round out to the edge of the plate. The instrument is currently being used to obtain a complete coverage of the Southern hemisphere down to $m_{v} \sim 11.5$ such that each plate, effectively $4^{\circ}$ square, has overlapping plates centred at each corner and at the middle of each side. In practice, this system of overlapping is applied to a number of zones in declination each about $10^{\circ}$ broad. Each plate is taken within $30^{\mathrm{m}}$ of the meridian and is given two $3^{\mathrm{m}}$ exposures above and below the 1950 standard positions, the geometrical plate centre being estimated to be within $20^{\prime \prime}$ of the tangential point. At the time of writing, coverage of the zone $-30^{\circ}$ to $-52^{\circ}$ is almost complete, and preparations for the zone $0^{\circ}$ to $-30^{\circ}$ well advanced.

\section{Outline of Technique}

The scheme of analysis proposed is to correct the relative coordinates of stars on each plate for such effects as (a) aberration, (b) refraction, (c) optical aberration, and (d) significant relative proper motions, on the assumption that these effects are either calculable to a first order or constant from plate to plate, and then to adjust the configuration of stars in common to each pair of overlapping plates for scale and orientation, so that the relative displacements on the celestial sphere of the stars in common are minimized. The relative displacement in spherical coordinates of each pair of plate centres is thus calculated. A large system of overlaps produces a network of such displacements which are then adjusted subject to the overriding condition that this network lies exactly on the celestial sphere. 
Suppose that the centres of two neighbouring overlapping plates can be fixed relative to each other with an accuracy $e$ (standard error) in either coordinate, then two centres $180^{\circ}$ apart on the celestial sphere can be fixed with an accuracy of $e \sqrt{n}$, where $n$ is the number of overlaps joining them along a great circle. There are in the limit, $2 n$ independent series of overlaps joining two such centres in a whole sky coverage, although in practice, there is a degree of correlation near the centres in question due to the finite size of the photographic plates. Two centres $180^{\circ}$ apart may thus be fixed relative to each other with an accuracy of the order of $e(\sim e \sqrt{n} / \sqrt{2 n})$, equal to the accuracy of a single overlap. The practical realisation of a system of relative star positions from overlapping photographic plates may thus be reduced to one of making a single overlap as precise as possible. If $m$ stars are common to each of two overlapping plates and each relative star position can be determined with an average accuracy of $\sigma$, the relative centres can be fixed with an accuracy of $e \sim \sigma(2 / \mathrm{m})^{\frac{1}{2}}$. By way of example, with the $20-\mathrm{cm}$ camera, it may be expected that $\sigma \sim 1 \mu \approx 0$ ". 1 , so that with 8 stars per half plate we anticipate $e \sim 0.05$, easily comparable and potentially better than the typical systematic positional accuracy associated with other instruments.

Such an order of accuracy approaches the random dispersion of proper motions associated with the stars used in these overlaps so that it is important to complete the photographic cover of the sky in as short a time as possible, or to regard stellar proper motion as an unknown to be sought in the analysis. In the present work, however, we shall without introducing any great error, consider the simple case where all the plates are assumed to be obtained simultaneously at one epoch. In order to refer such a rigid system of relative star positions to an inertial frame, it is necessary to obtain the positions of only a very few star-like extragalactic objects or suitably dimmed 'fundamental' stars on some overlapping plates. The former alternative seems the most promising and is to be investigated with the Cape instrument.

\section{Theory of Analysis of Overlapping Photographic Plates}

The plates obtained with the Cape camera have typically some 150 star images per exposure. Available facilities and personnel make it impossible to measure the coordinates of all these images, and the preliminary investigations are restricted to measurement of stars in the S.R.S. programme - with a density of about 1 per square degree. For each of these stars, provisional equatorial coordinates are available, and in the following discussion these are used as a first approximation to the star positions. It should be understood, however, that these are used only for convenience and that there is no fundamental need for them.

In the first instance, measured coordinates for each 'plate-star' $(p, q)$ are obtained relative to an arbitrary origin chosen to be as near to the tangent point as possible. Then using an assumed focal length, these coordinates are corrected for such cal- 
culable effects as differential aberration, differential refraction, and optical distortion to produce 'observed coordinates' $\left(x_{1}, x_{2}\right)$. It is recognized that these corrections cannot be calculated exactly since small unknown changes in the focal length $\left(x_{3}\right)$, the atmospheric conditions, and the optical characteristics of the telescope may occur. It may reasonably be expected, however, that our estimates of these corrections differ from the 'real' corrections by second-order amounts which are linearly related to $x$ and $y$.

We now consider all the plate-stars appearing on one plate and form the quantities

$$
c_{i}=\left(\Sigma_{i} x_{i}^{2}\right)^{-1 / 2} x_{i} \quad(i=1 \cdot 3)
$$

for each star, where $x_{3}$ is regarded as an 'observed focal length' and is in the first instance put equal to $x_{3}^{0}$. The following transformation relates $c_{i}(i=1 \cdot 3)$ to the star's equatorial direction cosines $\tau_{j}(j=1 \cdot 3)$

$$
c_{i}=\Sigma_{j}\left\{\Sigma_{k}\left(\Sigma_{l} \alpha_{k l} \tau_{l}\right)^{2}\right\}^{-1 / 2} \alpha_{i j} \tau_{j} \quad(i, j, k, l=1 \cdot 3),
$$

in which $\left|\alpha_{i j}\right|$ represents a transformation peculiar to the plate concerned.

In terms of the familiar plate constants for differential scale, orientation, and displacement of the origin,

$$
\left|\alpha_{i j}\right| \equiv\left|\begin{array}{ccc}
1+a & b & c \\
d & 1+e & f \\
0 & 0 & 1
\end{array}\right| \quad\left|\begin{array}{rrrrr}
-\sin \alpha_{0} & & \cos \alpha_{0} & 0 \\
-\sin \delta_{0} & \cos \alpha_{0} & -\sin \delta_{0} & \sin \alpha_{0} & \cos \delta_{0} \\
\cos \delta_{0} & \cos \alpha_{0} & \cos \delta_{0} & \sin \alpha_{0} & \sin \delta_{0}
\end{array}\right|
$$

where $\left(\alpha_{0}, \delta_{0}\right)$ are the coordinates of the tangent point.

The procedure followed is to solve Equation (2) for $\left|\alpha_{i j}\right|$ iteratively using adopted direction cosines $\tau_{j}$ (e.g. corresponding, at first, to the provisional equatorial coordinates) for each plate-star, subject to the condition that

$$
\Sigma_{j} \alpha_{3 j}^{2}=1 \quad(j=1 \cdot 3) \text {. }
$$

Then, with these values of $\left|\alpha_{i j}\right|$ and the final values of $c_{i}$ in Equation (1), we derive improved values for $\tau_{j}$ for each plate-star with corresponding weight $w$.

Weighted means of $\tau_{j}$ are then formed from overlapping plates on which each star appears, and these are then used as improved estimates in repeating the above procedure. The convergence of this process has not yet been adequately tested, but in principle, it is possible for star positions at the pole to affect those at the equator, thus necessitating at least 5 iterations. In practice, it is likely that such effects will be submerged by the measuring and plate errors.

The only restriction placed upon this method of analysis is in the first iteration. The provisional equatorial coordinates are probably bad enough to produce unnecessarily inaccurate estimate of $\left|\alpha_{i j}\right|$ and by implication, inaccurate estimates of the coordinates of each tangent point, $\left(\alpha_{0}, \delta_{0}\right)$. We regard the telescope system and measuring machine as sufficiently rigid to render the tangent point determinate within 
about $1^{\prime}$ and therefore apply the initial condition that $\alpha_{0}$ and $\delta_{0}$ are known, and solve Equation (2) for the inverted matrix $\left|\alpha_{k l}\right|^{-1}$ using $c_{i}$ as coefficients of the unknowns, since these are, at first, known with greater precision than $\tau_{j}$.

A FORTRAN programme for this routine has been prepared for use on the ICT 1900 machine at Herstmonceux. Basically, each iteration involves the processing of an unformatted magnetic tape to produce a revised but otherwise identical tape containing a series of records in which each successive set of records pertains to one plate. Each record contains 3 integer numbers ( $s=$ serial number on the tape, $p$, and $q$ if appropriate) and 9 non-integer numbers $\left(r_{k}(k=1 \cdot 9)\right)$. Each set of records contains (1) an initial master record in which $\left|r_{k}\right|=\left|\alpha_{i j}\right|$, the transformation peculiar to the plate; (2) a series of star records in which $r_{k=1.3}=x_{i=1 \cdot 3} ; r_{k=4.6}=\tau_{j=1.3} ; r_{k=7}=$ $w$; and (3) a terminator record for the plate concerned, in which $r_{k}$ has no significance. Figure 1 illustrates the nature of the reduction routine followed.

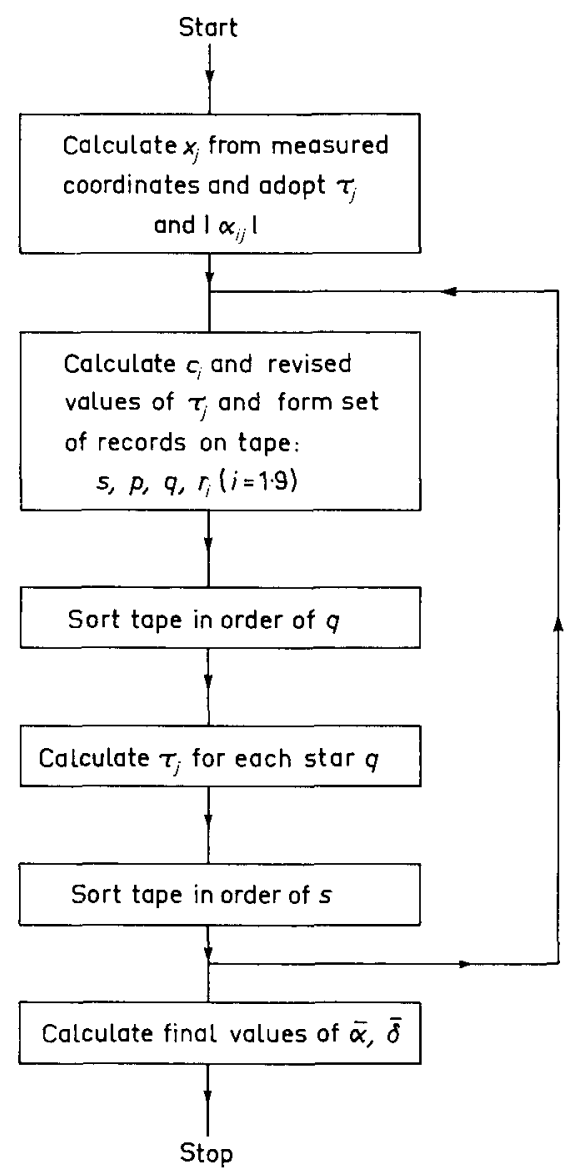

FIG. 1. 


\section{Current Investigations}

At the present, this programme of measurement and reduction is in the development stage, and at the Cape we are engaged on two preliminary investigations. The first is to test both for size and constancy, the effects on the relative star positions induced by the telescope-filter combination and by the star colours and magnitudes and by plate-emulsion irregularities. This is involving an examination of overlapping plates in the Cape E-regions, where accurate photometry is available for fainter stars. The second investigation is to test the predictions of the second section using a series of plates at constant declination in the Cape astrographic zone. Ultimately, it is hoped that this procedure will be extended to plates over the whole of the Southern hemisphere.

\section{Acknowledgements}

This investigation was suggested to me by Dr. R.H. Stoy. The organization of the observing programme is arranged by Mr. J.v. B. Lourens and Mr. T. W. Russo, who, with other members of the Cape Staff, are carrying out the plate measurement. I would like to thank Mr. C. A. Murray and Mr. W. Nicholson for discussions which clarified the technique to be used, and for help in writing the reduction programmes. 